\title{
Multiple shell shower fronts in EAS with ARGO-YBJ
}

\author{
G. Marsella ${ }^{\mathrm{a}}$ on behalf of ARGO-YBJ Collaboration
}

Universitá del Salento and INFN Lecce

\begin{abstract}
The ARGO-YBJ experiment is an Extensive Air Shower array that has been operated at the high altitude Yangbajing Cosmic Ray Laboratory (Tibet, P.R. China 4300 m a.s.1.) in its final configuration since December 2007 until February 2013. The detector consists of a dense layer of Resistive Plate Counters (RPCs) covering an area of about $11000 \mathrm{~m}^{2}$. It has been designed to measure the temporal and spatial structure of Extensive Air Showers (EAS) with high space-time resolution. The detector gives a quite highly detailed picture of shower footprints at ground. It is perfectly suitable to understand the EAS morphology. These detector characteristics have been used for seeking particles of large rest mass produced in cosmic rays by measuring the Multiple Shell Shower Fronts relative delays. The technique and preliminary results will be illustrated in the present work.
\end{abstract}

\section{Introduction}

The ARGO-YBJ experiment (Astrophysical Radiation with Ground-based Observatory at YangBajing) has been designed to study cosmic rays and cosmic $\gamma$-radiation at energy larger than few hundreds $\mathrm{GeV}$, by detecting air showers at high altitude with wide-aperture and high duty cycle. The apparatus is a single layer detector logically divided into 153 units called clusters $\left(7.6 \times 5.7 \mathrm{~m}^{2}\right)$, each made of 12 Resistive Plate Counters (RPCs) operating in streamer mode. Each RPC is read out using 10 pads $\left(61.8 \times 55.6 \mathrm{~cm}^{2}\right)$, which are further divided into 8 pick-up strips providing a larger particle counting dynamic range. The signal coming from all the strips of a given pad are sent to the same channel of a multi-hit TDC, which can record up to 16 signals on the same pad but with a time separation of at least $90 \mathrm{~ns}$, while the strip multiplicity (up to 8 on the same pad) could be recorded without deadtime. Pads are the time elemental units for measuring the pattern of the shower front with time resolution of $\sim 1.8 \mathrm{~ns}$ and the limitation that during $90 \mathrm{~ns}$, for each pad, only the first hit can be recorded, while the multiplicity can be saturated without time limitations. This aspect limits the time study in a very dense area of the shower such as few meters around the shower core. The shower time is defined as the time difference between the starting of TDC counting, when a particle hits the detector, and the common stop signal stated by the occurrence of the trigger condition in a time window of $2 \mu \mathrm{s}$. The apparatus covers an area of $74 \times 78 \mathrm{~m}^{2}$ with $92 \%$ of active area in the central array. To improve the reconstruction capability, the surrounding area has been partially instrumented with a guard ring of RPCs extending the detector layout up to $100 \times 110 \mathrm{~m}^{2}$. ARGO-YBJ allows a complete and detailed three-dimensional reconstruction of the shower front with unprecedented spatial and time resolution.[1-4].

\footnotetext{
a e-mail: giovanni.marsella@le.infn.it
}

\section{Time structures}

A flat array like ARGO-YBJ can measure the particles arrival times and their densities at ground. The digital readout allows detecting shower secondary particles down to very low density and the high space-time granularity is able to provide a fine sampling of the shower front close to the core. The time profile of the shower front can be reconstructed by the time of fired pads. Particles within several tens of meters from the shower core, due to geometric considerations on the principal physical processes in the shower cascade developments, are expected to form a curved front. As a first approximation, to reconstruct the primary particle arrival direction, the space-time coordinates (position and time of fired pads in the event) can be fitted by a cone whose axis crosses the core position at ground. This choice allows simpler calculation algorithms fully efficient in reconstructing the principal shower characteristics such as direction and energy. The arrival times of the particles are fitted by minimizing the following quantity:

$$
S^{2}=\frac{1}{W} \sum_{i=1}^{N_{h i t}} w_{i}\left(t_{i}-t_{0}-\frac{x_{i}}{c} l-\frac{y_{i}}{c} m-\frac{R_{i}}{c} \alpha\right)^{2} .
$$

The reconstructed parameters are the direction cosines $l, m$ and the time $t_{0}$. The sum is over the fired pads, $W=$ $\sum_{i=1}^{N_{h i t}} w_{i}, w_{i}$ being the number of strips fired in the i-th pad, $t_{i}$ is the measured time, $x_{i}, y_{i}$ are the pad coordinates and $c$ is the light velocity. The conicity correction depends on the conicity coefficient $(\alpha)$ and on the pad distance $\left(R_{i}\right)$ to the core in the plane perpendicular to the shower axis (shower plane, see Fig. 1). A Maximum Likelihood based algorithm, using a NKG-like [5] lateral distribution function is used to perform a reliable reconstruction of the shower core position and arrival direction up to the edge of the active carpet and slightly beyond [6].

Events having large time residual RMS with respect to the shower front have been investigated. The longitudinal

This is an Open Access article distributed under the terms of the Creative Commons Attribution License 4.0, which permits unrestricted use, distribution, and reproduction in any medium, provided the original work is properly cited. 


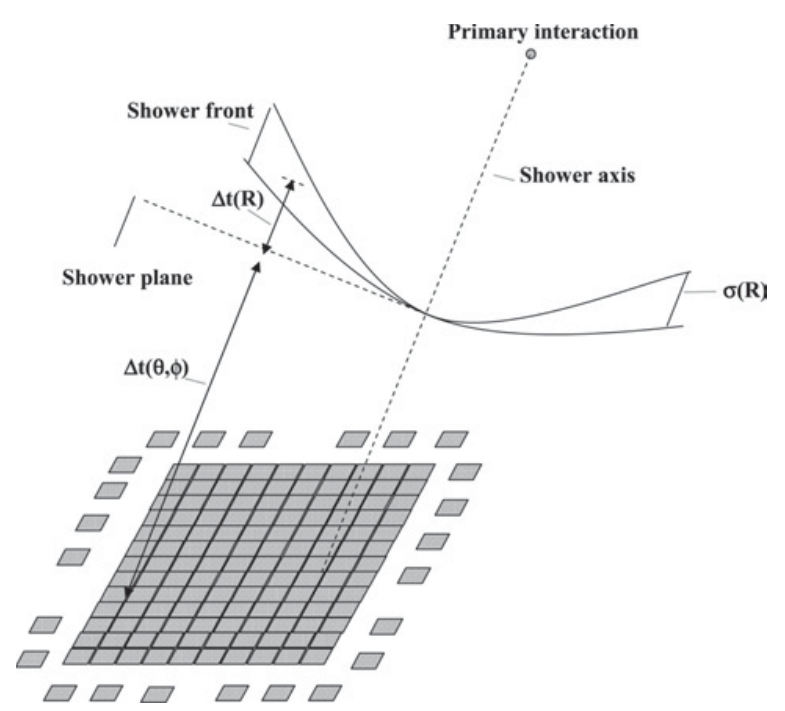

Figure 1. Sketch of shower front geometry and observables.

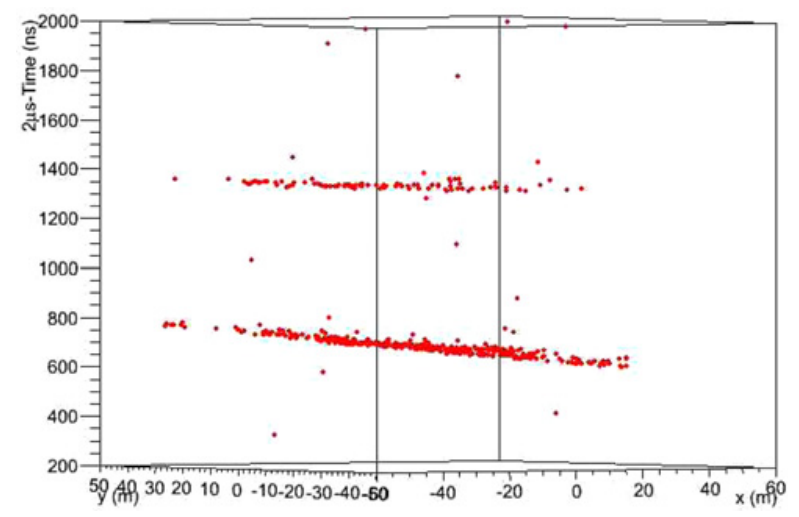

Figure 2. Multiple-Shell shower Front example. The threedimensional view of a MSF is shown. On the $\mathrm{z}$ axis the arrival time is plotted. It is defined as the time difference between the starting of TDC counting, when a particle hits the detector, and the common stop signal stated by the occurrence of the trigger condition in a time window of $2 \mu \mathrm{s}$. The two subshowers are evident and distant more than $300 \mathrm{~ns}$.

time structures in data could help to better define selection criteria for particular analysis, such as mass composition or "exotic" physics, and allow a better determination of EAS disc structure and correlations between front profile, front thickness and core distance. Several structures have been observed, but Multiple-Shell shower Fronts (MSF) case will be discussed.

\section{Multiple-Front showers}

In Fig. 2 the three-dimensional view of a MSF is shown. On the $\mathrm{z}$ axis $2 \mu \mathrm{s}$ minus the arrival time is plotted. In the past, many experiments detected anomalous delayed showers [7-10]. As a possible explanation many phenomena have been considered such as heavy particle production in the interaction of high energy particles on atmospheric nuclei and relative decay and the existence of tachyons $[11,12]$. Selection criteria were defined in order to reduce the accidental background events and a phase space was built in order to constraints the main characteristics of possible such heavy particles, in

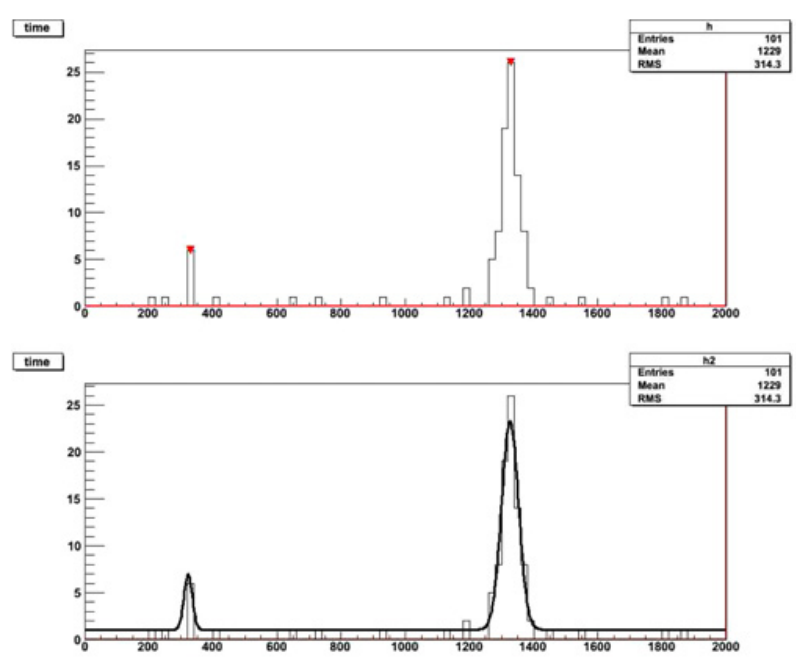

Figure 3. TSpectrum peak detection example. In the lower part the fit with a sum of 2 gaussian is shown.

particular the mass limits, decay time and transverse momentum.

\subsection{MSF selection}

To select the MSF, events with a multiplicity $N_{h i t}>$ 100 are requested. This is an arbitrary value to avoid sub events with a very little number of hits $\left(N_{h i t}>\right.$ $20)$ to get a sufficiently well reconstructed event and reduces the sample at $10 \%$. After that the ROOT function TSpectrum [13] is applied on the time distribution asking the detection of at least 2 peaks. The TSpectrum algorithm has been developed to detect peaks in spectrum analysis. Then a fit of the sum of 2 gaussians is applied using as starting parameters the returned ones by TSpectrum. Finally the time distance between the 2 selected peaks is requested to be greater than the sum of the relative evaluated RMS. This criteria is requested to be sure to detect 2 well separated peaks as shown in Fig. 3. Once a double peak event has been selected, the minimum of the time distribution between the two peaks is found and the hits are divided with respect to it detecting in this way the 2 sub showers.

To test the efficiency of the algorithm in selecting double showers, a sample of "artificial" double events has been generated using real events and putting in a single event two consecutive events where the time of all the hits of the second one have been shifted randomly by a time factor extracted by a flat distribution between $-700 \mathrm{~ns}$ and 1300 ns. 741000 events have been generated and an efficiency of $10.94 \pm 0.05 \%$ for the algorithm has been evaluated. In Fig. 4 the fraction of events detected in each bin is plotted in function of the absolute value of the delay time generated. Around the zero, which corresponds to showers arriving at the same time, the algorithm is unefficient because the two shower time peaks are not well separated.

The sample of artificial events is particularly useful in reproducing the random coincidence event distributions. In Fig. 5 the multiplicity distribution of the artificial double events is shown. 


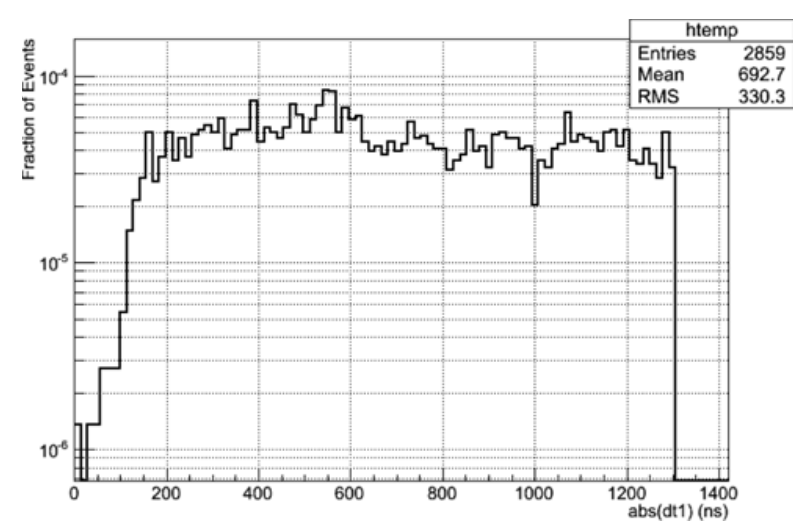

Figure 4. Fraction of events selected in function of the absolute value of the shift time of the second front in the "Artificial" MSF events generated by ordinary events.

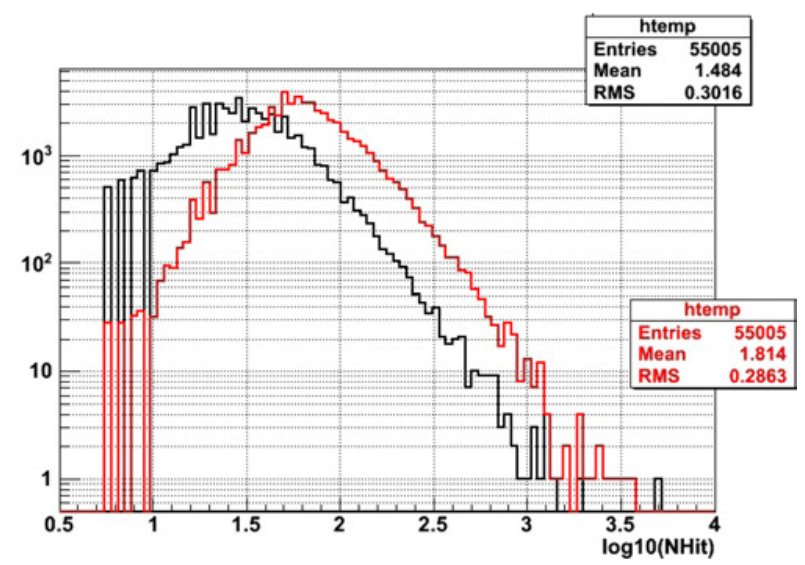

Figure 5. $N_{H i t}$ distribution of the artificial double events.

\section{Data analysis}

The selected double sub showers are reconstructed separately using a planar fit to get the direction and the likelihood method to reconstruct the core position. To determine the time distance between the sub showers 2 variables are available:

- a) the detected peak time distance;

- b) the time difference between the $T_{0}$ determined by the planar fits, which correspond to the time at which the shower planes cross the center of the carpet.

The 2 variables are well correlated as shown in Fig. 6. In the case of vertical showers the two variables are equivalent.

The data selected are mainly random coincidences of 2 showers in the DAQ time window. The expected number of such events can be calculated applying Poisson formula for time coincidences:

$$
\lambda_{\text {exp }}=\lambda_{1} \times \lambda_{2} \times \tau
$$

where $\lambda_{1}$ is the rate of shower events of type $1, \lambda_{2}$ is the rate of shower events of type 2 , corresponding to the first and the second shower respectively, and $\tau$ is the DAQ time window $(\tau=2 \mu \mathrm{s})$. The characteristics of the sub events are determined by the selection criteria and the reconstruction quality cuts applied in this analysis. In

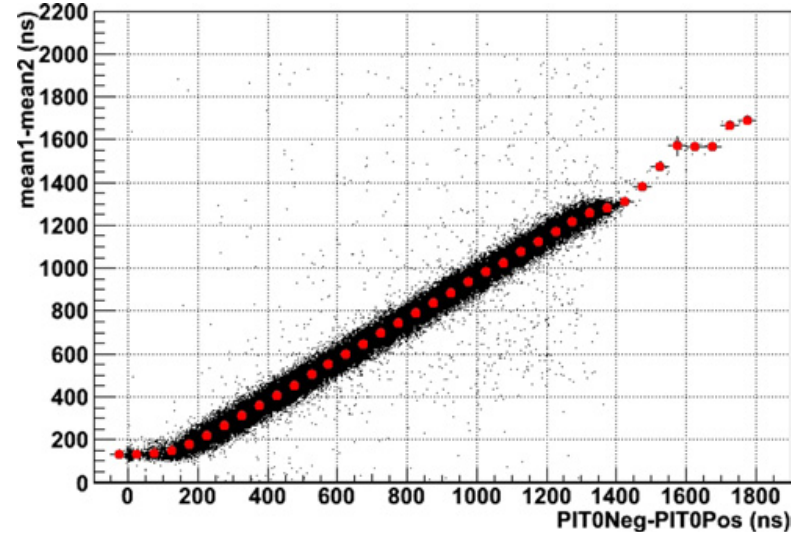

Figure 6. The scatter plot between the time peak and $T_{0}$ differences.

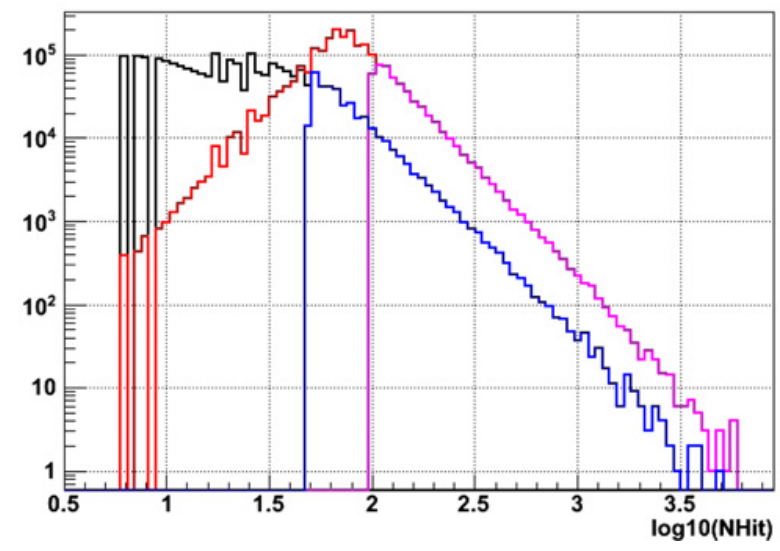

Figure 7. $N_{H i t}$ distribution of the detected sub showers. In red is the distribution of the first shower, in black the second shower. In Pink and blue the multiplicity cut in this analysis.

Fig. 7 the multiplicity distribution $N_{\text {nhit }}$ and the selection applied on that variable are shown: $N_{\text {nhit } 1}>100$ and $N_{\text {nhit } 2}>50$. A last cut is applied on both sub showers: It is requested that the reconstruction parameter $S^{2}<20 \mathrm{~ns}^{2}$. Starting from an acquisition rate of $3.27 \mathrm{kHz}$, for the selected events the following values have been obtained:

$$
\begin{aligned}
& \lambda_{1}=(0.419 \pm 0.001) \mathrm{kHz} ; \\
& \lambda_{2}=(1.152 \pm 0.002) \mathrm{kHz} ;
\end{aligned}
$$

giving an expected rate

$$
\lambda_{\text {exp }}=(0.957 \pm 0.003) \mathrm{Hz} .
$$

$3.05 \times 10^{9}$ events have been processed, $2.1 \times 10^{6}$ events have been selected as double coincidences and, among these, $99 \times 10^{3}$ events have been selected as double coincidences with quality cuts on sub showers (multiplicity and $S^{2}$ ). Taking in account the efficiency of the selection algorithm the observed rate is:

$$
\lambda_{o b s}=(0.974 \pm 0.008) \mathrm{Hz}
$$

(see Table 1)

Applying Feldman and Cousins [14] statistics an upper limit at $90 \%$ C.L. of $5.7 \times 10^{-5}$ as a fraction of the total 
Table 1. Expected and Observed rate

\begin{tabular}{ll}
\hline Expected rate & Observed rate \\
\hline $0.957 \pm 0.003 \mathrm{~Hz}$ & $0.974 \pm 0.008 \mathrm{~Hz}$ \\
\hline
\end{tabular}

events is obtained as generated by double showers not due to random coincidences but possibly correlated to a unique cosmic ray event. From this result is possible to obtain an upper limit to a possible flux of "Double Showers".

It has been demonstrated that these events are mainly due to accidental coincidences. Rate and other observable distributions are compatible with the expected one from pure accidental coincidences between different showers.

Due to the relatively small area, around $5800 \mathrm{~m}^{2}$ partially extended to $11000 \mathrm{~m}^{2}$ with the guard ring, and assuming a mean first interaction height of about $20 \mathrm{~km}$, the angular difference expected between two subshowers of common origin is less than $0.2^{\circ}$. In this case the two subshowers should have approximately the same development and no delay is expected. Late decay or interaction of a leading heavy particle could originate secondary showers with a wider angular difference, with a difference in the multiplicity distribution (late produced particle should develop showers with lower multiplicity) and "large" arrival time delay. In case of production of a massive particle with rest mass $\mathbf{M}$ and laboratory energy $\mathrm{E}$, produced at the top of the atmosphere, it will arrive at the detector level after traveling a distance $l$ with a time delay $\Delta t$ respect to the primary shower front given by:

$$
\Delta t=L(1 / v-1 / c) \approx L / 2 c \gamma^{2}
$$

where $\mathrm{L}$ is the distance from the point at which the particle has been produced and the height of decay or interaction, $v$ is the velocity of the massive particle, $c$ is the light velocity and $\gamma=E / M$. Assuming that the particle is early produced $(L \sim 20 \mathrm{~km}), \gamma$ ranges from 26 to 5 for time delays, $\Delta t$, ranging from $50 \mathrm{~ns}$ to $1.4 \mu \mathrm{s}$, which is the range of sensitivity to this analysis with the ARGOYBJ detector. Thus a particle of mass $M=10 \mathrm{GeV}$ with energy $E=100 \mathrm{GeV}(\gamma=10)$ produced $10 \mathrm{~km}$ above the detector would arrive $160 \mathrm{~ns}$ behind the leading shower. If it produces a shower, this could be detected as a secondary delayed shower. So geometrical and kinematic considerations allow to use the time difference $\Delta t$ between the arrival time of the subshowers to define constraints on heavy mass production in cosmic ray interactions $[15,16]$. From the distribution of the subshower time delay it is also possible to determine the decay time $\tau$ [17]. The decay probability of the particle as a function of $t$ is given by $P(t)=\exp [-t / \gamma \tau]$. By using the Eq. (7) we may express the decay time as $l / v=\Delta t /(1-\beta) \gamma \tau$. So the probability of the second shower to have a delay $\Delta t$ is given by the following

$$
P(\Delta t)=\frac{1}{(1-\beta) \gamma \tau} \exp [-\Delta t /(1-\beta) \gamma \tau] .
$$

The frequency is obtained by integrating the probability and is still proportional to $P(\Delta t)$

$$
f(\Delta t)=C \times \exp \left[-\Delta t / \Delta t_{0}\right]
$$

where $\Delta t_{0}=\tau / 2 \gamma$.

\section{Summary and conclusions}

ARGO-YBJ detector allows detailed studies of EAS spacetime features. Multiple Shell Shower fronts have been detected quite efficiently. They are mainly accidental coincidences due to the very high trigger rate as has been proved in this work. An upper limit on the fraction of double shell shower fronts, as a flag of possible "exotic" physic events in CR, has been presented.

It is possible to define more stringent analysis cuts based on the event geometry and on the particle interaction kinematics to reduce the large background. The huge mass of data of ARGO-YBJ will allow to investigate further details and rare events with good statistics.

\section{References}

[1] C. Bacci et al., NIM A 443 (2000) 342

[2] G. Aielli et al., NIM A 562 (2006) 92

[3] G. Aielli et al., NIM A 608 (2009) 246

[4] M. Iacovacci et al., Proc. 32nd ICRC (2011)

[5] K. Greisen, Ann.Rev.Nucl.Sci. 10 (1966) 63

[6] G. Di Sciascio et al., Proc. of 28th International Cosmic Ray Conference (ICRC 03), Tsukuba, Japan, Session OG.2.5 (2003) 3015

[7] M. Ambrosio et al., Astrop. Phys. 11 (1999) 437

[8] G. Damgaard et al., Phys. Lett. 17 (1965) 152

[9] B.K. Chatterjee et al., Proc. 9th ICRC (1965)

[10] L. W. Jones et al., Phys. Rev. 164 (1967) 1584

[11] G. Feinberg, Phys. Rev. 159 (1967) 1089

[12] L. W. Jones et al., Rev. Mod. Phys. 49 (1977) 717

[13] TSpectrum, http://root.cern.ch

[14] G.J.Feldman and R.D.Cousins, Phys. Rev. D 57 (1998) 3873-3889

[15] M. Yoshida et al., J. of the Phys. Soc. Jap. 53 (1984) 1983

[16] V. I. Yakovlev et al., Yadernaya Fizika 73 (2010) 816

[17] N. Sakuyama et al., Il Nuovo Cimento vol. 6 C (1983) 371 